\title{
Editorials
}

\section{Are official psychiatric classification systems for mental disorders suitable for use in primary care?}

The two major classifications for mental disorders, ICD-11 and the DSM-5, have recently been updated ${ }^{1,2}$ but it is not clear that either is suitable for routine use in primary health care $(\mathrm{PHC})$ settings. Mental disorders are described as though they are separate from one another, with clear borders between them, and within these borders are relatively homogeneous. In fact, neither of these is true, since there are unbroken continuums between them, and there is considerable heterogeneity within their borders. ${ }^{3}$ Both the ICD-11 and the DSM-5 make arbitrary divisions between the various overlapping syndromes of common mental disorders, and when more than one are present, treat them as though they are two quite separate illnesses. But in general medical settings, this type of comorbidity is the rule, not the exception. It is convenient for clinicians to use categorical models, but dimensional models fit the data far better when these are used with large data sets such as in the World Health Organization's (WHO) first major study in primary care, carried out using a research interview to detect common mental disorders in 14 different countries. ${ }^{4}$ The model of best fit is a dimensional model with a common general factor on which all symptoms load, and three subsidiary factors dealing with anxious, depressive, and somatic symptoms. ${ }^{5}$ Major depressive episode and dysthymic disorder also load on the depression factor; and somatisation disorder, hypochondriasis, and neurasthenia also load on the somatic factor. Anxious symptoms only loaded on the general factor; there was very little specific anxious variance on the anxiety factor.

\section{ANXIOUS AND DEPRESSIVE SYMPTOMS}

The most common psychological disorders in general medical practice consist of two highly correlated sets of symptoms anxious and depressive. The high correlation between them is because they both have high loads on the general factor. The DSM-5 system requires anxious symptoms to be present for 6 months, and the ICD11 system requires anxious symptoms for 'several months', but both systems require depressive symptoms for only 2 weeks. In primary care, it is important to know which symptoms are currently bothering the patient, and it is of interest to compare the conventional procedure, where anxious symptoms have to last 6 months, with a

many patients develop anxious symptoms simultaneously with depressive symptoms, but conventional diagnostic practices ignore anxious symptoms that have not lasted at least several months.

different procedure, where current anxiety' is recorded if anxious and depressive symptoms have lasted only 2 weeks. If the same time duration is required for each, the combination of both sets of symptoms becomes the most common disorder encountered in PHC settings, and is best thought of as 'anxious depression'. If such patients are compared with patients who have depression but not anxiety, then the frequency of 'depression only' drops from $4.7 \%$ to $2.3 \%$, while the frequency of "anxious depression' rises from $3.4 \%$ to $5.7 \%{ }^{6}{ }^{6}$ This is because many patients develop anxious symptoms simultaneously with depressive symptoms, but conventional diagnostic practices ignore anxious symptoms that have not lasted at least several months in ICD-11 (or 6 months in DSM-5). This is a definite shortcoming of the official classifications, because many people identified only as having 'major depressive episode' are quite likely to have anxious and depressive symptoms.

People with anxious and depressive symptoms have both a greater number and more severe depressive symptoms than individuals with non-anxious depressive symptoms; they are more likely to be suicidal and they have a more difficult future course than those with non-anxious depression. ${ }^{7}$ Parents of individuals with anxious depressive symptoms are more likely to have a range of mental disorders, including hypomania, whereas individuals with non-anxious depressive symptoms have raised rates only of depression in their family history. ${ }^{8}$ In the field trial that concentrated on anxious depression, the use of two 5-item scales - one for depression and the other for anxiety enabled GPs to make diagnoses of largely illiterate populations.

\section{CLASSIFICATIONS FOR PRIMARY HEALTH CARE SETTINGS}

Both of the major classifications for mental health specialists expect clinicians to administer a wide range of questions dealing with common mental disorders, and take the view that when requirements are satisfied for several different disorders, that these are 'comorbid' with one another. GPs are unlikely to want to see how many allegedly 'separate' mental disorders are present, and are often more concerned with detecting common mental disorders that indeed are comorbid with chronic physical disease, as well as to deal with multiple somatic symptoms that preoccupy the patient, and are often accompanied by anxious and depressive symptoms that cause substantial associated disability.

With the above differences in view, the WHO produced a revised classification adapted for use in PHC settings, and this has been brought up to date to accompany the latest revision of the ICD, now consisting of 28 disorders. The committee that produced this classification contained equal numbers of males and females, of psychiatrists who teach mental health skills to trainees in PHC, and GPs with a publication record in mental disorders. We were concerned that GPs would in future have names for the disorders that can be shared with their patients. So medically unexplained symptoms' became 'bodily stress syndrome' (BSS), and hypochondriasis became "health anxiety'. These terms are not only less pejorative, but are more likely to lead to a therapeutic dialogue with the patient. The classification also includes substance use disorders, eating disorders, and common sexual problems, as well as five disorders of childhood. It has to roughly relate to the disorders dealt with by the main ICD-11, and also deals with major disorders, including three psychotic disorders, dementia, and delirium.

Field trials of the revised classification have been carried out in five countries (Brazil, China, Mexico, Pakistan, and Spain), with special attention to disorders characterised by anxiety and depression, as well as bodily stress disorders and health anxiety. These 


\section{“... 'medically unexplained symptoms' became 'bodily stress syndrome' (BSS), and hypochondriasis became 'health anxiety'. These terms are not only less pejorative, but are more likely to lead to a therapeutic dialogue with the patient.}

disorders have been substantially revised, while most of the rest of the system for PHC had been field tested earlier. For the field trial dealing with anxiety and depression, primary care physicians used two short 5 -item scales dealing with the detection of anxious and depressive symptoms so that illnesses could be detected in populations with low levels of literacy. ${ }^{9}$ The field trial used these scales for anxiety and depression to provide a practical way for primary care physicians to evaluate the

\section{Box 1. Brief screening scales for depression and anxiety}

\section{Five-item depression scale Screening items:}

1. Have you been feeling depressed every day for the past 2 weeks?

2. During the past 2 weeks, have you experienced less interest or pleasure from activities?

IOnly asked if positive response to either screening item)

During the past 2 weeks,

3. Have you experienced poor concentration?

4. Have you experienced feelings of worthlessness?

5. Have you felt you wanted to die or had thoughts of death?

\section{Five-item anxiety scale}

\section{Screening items:}

1. Have you felt nervous or anxious during the past 2 weeks?

2. During the past 2 weeks, have you found that you are not able to control your worrying?

IOnly asked if positive response to either screening item)

During the past 2 weeks,

3. Have you had trouble relaxing?

4. Have you felt so restless it was hard to keep still?

5. Have you felt afraid that something awful might happen? likelihood of mood and anxiety disorders without paper and pencil measures that are not feasible in many settings. These scales may provide substantially improved case detection, as compared to current primary care practice, and a realistic alternative to more complex diagnostic algorithms used with populations with low levels of literacy. ${ }^{10}$ They also confirmed that anxious depression is the most common disorder in each setting

Fink and his colleagues showed that if three or more somatic symptoms are present, patients with a range of common disorders with 'functional' symptoms can be identified in both primary care and general medical settings. ${ }^{11}$ A separate study showed that BSS is readily identified in all five countries, with over $70 \%$ identified as having both BSS and health anxiety, and with patients having an average of 10.9 different symptoms. ${ }^{12}$ The majority of patients with BSS had co-occurring diagnoses of depression, anxiety, or both. Anxious depression was the most common comorbid psychological disorder associated with the greatest disability. ${ }^{12}$

In parts of the world with high rates of illiteracy, GPs need to have a quick way of being alerted to psychological distress, and the two 5-item scales enable them to do that (Box 1). The demonstration that BSS occurs in all five countries and is strongly linked to anxiety and depression presents GPs with new data about patients who need careful management, and brings several different functional syndromes under a common name.

\section{Sir David Goldberg,}

Professor Emeritus, Institute of Psychiatry, Kings College London, London.

\section{Provenance}

Freely submitted; externally peer reviewed.

DOI: https://doi.org/10.3399/bjgp19X701369

\section{ADDRESS FOR CORRESPONDENCE}

\section{Sir David Goldberg}

Institute of Psychiatry, Psychology \& Neuroscience, Kings College London, 16 De Crespigny Park London SE5 8AF, UK.

Email: davidpgoldberg囚yahoo.com

\section{REFERENCES}

1. The World Health Organization. ICD-11: International Classification of Diseases for Mortality and Morbidity Statistics. 11th edn Geneva: The World Health Organization, 2018. https://icd.who.int/browse11/l-m/en laccessed 4 Jan 2019).

2. American Psychiatric Association. Diagnostic and Statistical Manual of Mental Disorders, (DSM-5). 5th edn. Washington: APA, 2013.

3. Goldberg D. The heterogeneity of "major depression". World Psychiatry 2011; 10(3): 226-228

4. Ustün TB, Sartorius N (eds). Mental illness in general health care: an international study. Chichester: Wiley-Blackwell, 1995.

5. Simms LJ, Prisciandaro JJ, Krueger RF, Goldberg DP. The structure of depression, anxiety and somatic symptoms in primary care. Psychol Med 2012; 42(1): 15-28.

6. Goldberg D, Simms LJ, Gater R, Krueger RF. Integration of dimensional spectra for Depression and Anxiety into Categorical Diagnoses for General Medical Practice. In: Regier DA, Narrow WE, Kuhl EA, Kupfer DJ. eds. The Conceptual Evolution of DSM-5. Washington: American Psychiatric Publishing, 2011.

7. Goldberg D, Fawcett J. The importance of anxiety in both major depression and bipolar disorder. Depress Anxiety 2012; 29(6): 471-478.

8. Goldberg DP, Wittchen HU, Zimmermann P, et al. Anxious and non-anxious forms of major depression: familial, personality and symptom characteristics. Psychol Med 2014; 44(6): 1223-1234.

9. Goldberg DP, Prisciandaro JJ., Williams P. The primary health care version of ICD-11: The detection of common mental disorders in general medical settings. Gen Hosp Psychiatry 2012; 34(6): 665-670.

10. Goldberg DP, Reed GM, Robles R, et al. Screening for anxiety, depression, and anxious depression in primary care: A field study for ICD-11 PHC. J Affect Disord 2017; 213: 199-206.

11. Fink P, Schröder A. One single diagnosis, bodily distress syndrome, succeeded to capture 10 diagnostic categories of functional somatic syndromes and somatoform disorders. $J$ Psychosom Res 2010; 68(5): 415-426.

12. Goldberg DP, Reed GM, Robles R, et al. Multiple somatic symptoms in primary care: A field study for ICD-11 PHC. WHO's revised classification of mental disorders in primary care settings. J Psychosom Res 2016; 91: 48-54. 